\title{
CALIDAD TOTAL: UNA ALTERNATIVA DE GESTIÓN PARA EL DESARROLLO DE LA PRODUCCIÓN CIENTÍFICA EN LA EDUCACION SUPERIOR
}

\author{
TOTAL QUALITY: AN ALTERNATIVE OF MANAGEMENT FOR THE \\ DEVELOPMENT OF SCIENTIFIC PRODUCTION IN HIGHER EDUCATION
}

\author{
Verónica López Lara, Mgs. \\ Magíster en Administración de Empresas (Ecuador). \\ Docente de investigación de la Universidad Técnica del Norte, Ecuador. \\ vdlopez@utn.edu.ec \\ vemelop@hotmail.com
}

Jesús Antonio Gómez Escorcha, PhD.

Doctor en Ciencias de la Educación (Venezuela). Docente tiempo completo, Universidad de Otavalo, Ecuador. jgomez@uotavalo.edu.ec; jesgomez35@gmail.com

\section{ARTÍCULO DE INVESTIGACIÓN}

Recibido: 5 de marzo de 2018

Aceptado: 19 de marzo de 2018

\section{RESUMEN}

El objetivo principal del estudio fue implementar acciones formativas referentes a la calidad total como alternativa de gestión para el desarrollo de la producción científica en educación superior. Por consiguiente, se fundamentó en el paradigma cualitativo, modalidad Investigación Acción Participativa (IAP), se organizó a través del diagnóstico, planificación que permitió desarrollar la metodología con la finalidad de proporcionar la solución al problema planteado. La recolección de evidencias, se utilizó la observación directa, mediante el instrumento de guion de observación y la guía de entrevista orientada. El procedimiento empleado en la selección de los informantes, fue el muestreo no probabilístico, tipo intencional. La validez y la fiabilidad, se fundamentó en la categorización y teorización través de la matriz de triangulación. Como resultado, se logró desarrollar acciones que permitieron orientar al docente sobre la calidad total como medio 
que mejoró la gestión en la producción científica. Se concluyó que el uso de las herramientas de la calidad total tales como; Gráficos de Control, Diagrama Causa - Efecto, Histograma, Diagrama de Pareto, Diagrama de Dispersión y la Estratificación, facilitó perfeccionar el proceso de investigación y de gestión. Además, el manejo de los lineamientos de la ISO 900, el cumplimiento de los indicadores establecidos por el CEAACES y el uso de la base datos Scimago Journal \& Country Rank, Elsevier, permitieron la publicación de textos como parte de la gestión en la producción científica en la educación superior.

Palabras clave: calidad total, gestión, desarrollo, producción científica, educación superior.

\section{ABSTRACT}

The main objective of the study was to implement training actions regarding total quality as an alternative management for the development of scientific production in higher education. Therefore, it was based on the qualitative paradigm, Participatory Action Research (IAP) modality, was organized through the diagnosis, planning that allowed to develop the methodology in order to provide the solution to the problem posed. Evidence collection, direct observation was used, through the observation script tool and the guided interview guide. The procedure used in the selection of informants was non-probabilistic sampling, intentional type. Validity and reliability were based on categorization and theorization through the triangulation matrix. As a result, it was possible to develop actions that allowed the teacher to be guided on total quality as a means to improve the management of scientific production. It was concluded that the use of total quality tools such as; Control Charts, Cause Diagram - Effect, Histogram, Pareto Diagram, Scatter Diagram and Stratification, facilitated perfecting the research and management process. In addition, the management of the ISO 900 guidelines, compliance with the indicators established by CEAACES and the use of the database Scimago Journal \& Country Rank, Elsevier, allowed the publication of texts as part of the management in scientific production in higher education.

Keywords: total quality, management, development, scientific production, higher education.

\section{INTRODUCCIÓN}

Hoy día la educación superior, concibe a la Universidad como una institución destinada a la enseñanza superior, es aquella que proporciona conocimientos especializados en diferentes áreas del saber, está estructuradas en varias facultades, divisiones, coordinaciones y 
unidades académicas que cumplen funciones específicas que concede los grados académicos, la divulgación científica y la generación de nuevos conocimientos que contribuyen con el desarrollo integral del ser humano. Por ello, la Universidad como organización, diariamente busca mantener un ambiente en el que las personas, trabajando en grupo promuevan el avance científico, económico, social que benefician a la comunidad y al colectivo en general.

Como antecedente de la investigación según el Instituto de Tecnología Massachusetts (MIT) "publicó el ránking de las 1000 mejores universidades del mundo; dos ellas pertenecen al Ecuador" (El Comercio, 2017, p.12) el instituto (MIT) expreso que en los primeros lugares se encuentra la Universidad de Stanford California a nivel mundial, en el Ecuador la Universidad San Francisco de Quito, se ubica entre los lugares 701 a 750 se halla como la universidad 57 del ránking y la Pontificia Universidad Católica del Ecuador que ocupa el lugar de 801 a 1000, puesto 78 a nivel de las universidades latinoamericanas. El instituto (MIT) lleva seis años consecuentes realizando este tipo de estudio fundamentado en criterios de evaluación de calidad, pertinentes en las áreas de investigación, producción científica, academia y vinculación.

Por tanto, la Universidad como organización educativa, es la encargada de promover la formación educativa a nivel superior, el progreso científico de la nación, ella cuenta con un grupo numeroso de personas profesionales que poseen las competencias necesarias para alcanzar en la población estudiantil una formación académica, basada en valores, aptitudes, capacidades especializadas para desempeñar a futuro una profesión con base a los resultados del nuevo conocimiento. Por ello, la Universidad Autónoma de Madrid (UAM) expresa "La producción científica, es el resultado de la investigación que llevan a cabo por cualquier medio y se recoge a través de soportes escritos, la universidad difunde el desarrollo investigativo para contribuir al crecimiento y evolución de la ciencia" (UAM, 2017, p. 1).

En efecto, el presente artículo tiene como objetivo principal, establecer la calidad total como alternativa de gestión para el desarrollo de la producción científica en la educación superior. La universidad ejerce una función muy importante para el desarrollo humano y científico del país. Ellas promueven la producción científica, la capacitación, la formación personal, el liderazgo basado en competencia, la creación de políticas educativas que contribuyen con la creación de una cultura científica en beneficio de la colectividad y de la nación. Por consiguiente, "las universidades de investigación suelen dar un énfasis especialmente al producto investigativo, pues cuentan con la expectativa de que sus académicos puedan consolidarse como líderes nacionales e internacionales en cada 
áreas de conocimiento" (Leisyte y De Boer, 2009, p. 12), la calidad total, ejerce una función importante en el logro de este objetivo, ella se encarga de establecer parámetros de calidad, estándares internacionales, que permiten a la organización cumplir con los objetivos previstos de manera eficiente y oportuna, satisfacer las necesidades establecidas, en otras palabras, la calidad total, es la prevención de los problemas, logro de objetivos de manera eficiente, crear un clima organizacional que responda rápidamente a las necesidades y requerimientos del cliente interno o externo.

En tal sentido, las políticas educativas se interesan en que las instituciones de educación superior mejoren sus resultados en producción científica a fin de cumplir con los indicadores de calidad establecido por los entes reguladores, tal es el caso, el Consejo de Evaluación, Acreditación y Aseguramiento de la Calidad de la Educación Superior, (CEAACES, 2017) expresa que los indicadores de calidad para la gestión de la producción científica no muestran resultados aceptables según la cuantificación de libros, artículos, ensayos, publicados en revistas indexadas en ISI wed de Knowledge, Scopus, Dialnet (Ecuador y Pazmiño 2014).

Con base en este hecho, las herramientas básicas de calidad total juegan un papel importante para desarrollar en el docente competencias para abordar estudios sobre fenómenos, realidades, investigaciones a fin de brindar nuevos aportes al campo de la ciencia y del conocimiento científico. El uso del Diagrama de Pareto, Histograma, Diagrama Causa Efecto, Gráficos de Control, Diagrama de Dispersión y la Estratificación, (Talavera, 2012) constituyen un grupo de herramientas que facilitan la generación de un número de ideas que se pueden convertir en soluciones sobre una temática específica. La problemática antes descrita, permite formular como pregunta de investigación la siguiente; ¿Cómo garantizar a través de la calidad total el desarrollo de la producción científica en la educación superior?

Sin embargo, la educación superior busca permanentemente el mejoramiento de sus procesos, implantando la calidad total como medio de gestión enfocado hacia el desarrollo de competencias investigativas dirigidas hacia el personal docente como elemento esencial para alcanzar excelencia educativa. En consecuencia, el enfoque de la calidad total, ofrece ventajas mediante el uso de herramientas que ayudan en la solución de realidades empíricas. Las cuales radican en la capacidad para ser utilizadas en un amplio rango de problemas relacionados con el control de calidad en el ámbito socioeducativo. 
Por tanto, "la preocupación por la calidad, eficiencia, productividad y competitividad que existe actualmente en todos los ámbitos del sistema educativo, es el resultado del proceso de globalización" (Cardoso y Cerecedo, 2012, p. 33). Por ello, esta situación se ha convertido en una necesidad para las instituciones educativas superior para lograr el desarrollo humano y científico de la sociedad. A partir de este contexto, la investigación se plantea interrogantes con el propósito de dar respuesta a la problemática seleccionada; ¿Cuál es la situación actual sobre la producción científica en la educación superior? ¿Cuáles son las herramientas de la calidad total que faciliten la gestión para el desarrollo de la producción científica?, por último, ¿Cuáles son las acciones sustentadas en la calidad total que permita gestionar el desarrollo de la producción científica a nivel de educación superior?

En consecuencia, la producción científica representa el resultado de la práctica diaria que ejerce el docente mediante la indagación, la revisión bibliográfica, la observación sobre fenómenos que pueden ser considerados como objeto de estudio, esto con el fin de generar un aporte teórico al campo del conocimiento. De allí, las políticas, las instituciones de educación superior, exigen establecer procesos sustantivos de producción que incluyen la docencia, investigación, la vinculación con la colectividad a fin de elevar la calidad institucional, el conocimiento científico y tecnológico.

\section{Objetivos de la Investigación}

Como objetivo general: Establecer la calidad total como alternativa de gestión para el desarrollo de la producción científica en la educación superior a nivel de la provincia de Imbabura. Asimismo, como objetivos específicos: analizar el contexto existe en correlación a la producción científica en instituciones universitarias de educación superior, establecer las herramientas básicas de calidad total como alternativa de gestión para el desarrollo de la producción científica y, por último, planificar acciones referentes a la calidad total como alternativa de gestión para el desarrollo de la producción científica.

\section{REVISIÓN TEÓRICA}

Calidad total como proceso de mejora continúa. 
La calidad total en la educación superior universitaria, está relacionada con distintas dimensiones y principios educativos que conforma la calidad del sistema educativo, "la calidad es una forma de dirigir la organización, agregando que el control de calidad es mucho más que una agrupación de proyectos técnicos y actividades de motivación, el control es una nueva manera de pensar en la dirección" (Camisón, Cruz y González, 2006, p. 51) se trata de crear un medio ambiente en la organización universitaria que responda de manera oportuna a las necesidades y requerimientos en el campo científico. Por eso, los miembros de la institución, deben valorar la función y el rol dentro de este proceso educativo en la educación superior a fin de elevar la producción científica.

Ahora bien, la administración de la calidad total, se focaliza en la necesidad y en la mejora continua de los procesos de la organización para la creación de nuevos conocimientos en las distintas áreas del saber, "el liderazgo, corresponde a la alta dirección fijar el rumbo en el que se moverá la organización, el cual va encaminado a crear unos valores claramente enfocados hacia el cliente" (Carro y González, 2012, p. 23).

En efecto, el docente de educación superior, ejerce un liderazgo de calidad con base a competencias que contribuyen a mejorar en la institución universitaria la divulgación del conocimiento científico, la investigación y la producción que contribuyen con el desarrollo humano. Este enfoque significa que este será quien juzgue la calidad, el comportamiento, el aprendizaje y el clima organizacional en la institución universitaria, a su vez, la mejora debe ser parte del trabajo diario de todas las áreas y departamentos de la organización. Para ello se deberá contar con las ideas de los empleados, los resultados de investigación, las sugerencias y opiniones de las personas.

Las entidades generadoras de conocimiento son llamadas a crear sus propios repositorios de conocimiento, sin embargo, es fundamental tener en mente paradigmas que se sustenten en rigurosos parámetros de calidad científica y estándares de gestión de información tanto local como internacional (Molina, Gómez, Barbosa y Cabrera, 2015, p. 40). 
Al respecto, la universidad como entidad generadora de conocimiento, representa una institución de educación superior que promueve la enseñanza, la profesionalización y la gestión en la investigación científica. Este hecho, es un proceso permanente evidenciado mediante la creación de libros, artículos publicados en revistas indexadas que proporcionan aportes teóricos para la construcción de conocimientos nuevos. En otras palabras, es función de la organización universitaria, emplear parámetros de calidad científica, estándares sustentados en procesos de indagación sistemática, al contraste empírico de las teorías, esto permitirá explorar nuevos campos del saber humano promover la enseñanza superior, el aprendizaje académico que contribuye con el desarrollo humano y la formación educativa. Las herramientas básicas de calidad total que faciliten al docente la gestión en la producción del conocimiento científico, expresa lo siguiente:

Las siete herramientas básicas de la calidad, pueden ser descritas genéricamente como métodos para la mejora continua y la solución de problemas, las siete herramientas básicas de la calidad son: Diagrama Causa - Efecto, Hoja de Comprobación, Gráficos de Control, Histograma, Diagrama de Pareto, Diagrama de Dispersión y la Estratificación (Talavera, 2012, p. 2).

En referencia a este planteamiento, las herramientas básicas de calidad total, representan un procedimiento metodológico que consiste en diagnosticar realidades empíricas de manera acertada y apropiada, visto como un proceso que incluye la relación entre el sujeto y objeto de estudio de una investigación. En otras palabras, estas herramientas, ayudan al docente como investigador a identificar, clasificar las posibles causas, efectos de un problema específico, establecer el registro de datos, informaciones, mediante herramientas estadísticas para controlar y mejorar el proceso de análisis de interpretación de los datos. Como también, contribuye a identificar la relación entre dos o más variables en una investigación de forma integral, en diferentes contextos, educativos, social y laboral.

\section{La gestión de la producción científica en educación superior.}

La gestión del conocimiento, puede ser definida como la forma de dirección orientada a la maximización del rendimiento del capital intelectual, entendido este como el conjunto de competencias institucionales distintivas, de carácter intangible, que 
permiten crear ventajas para la obtención de presupuestos mediante la colaboración comprometida de su comunidad y el uso de procesos en la producción, transmisión y transferencia de un conocimiento innovador, "La organización debe propiciar mecanismos que activen la dimensión colectiva del conocimiento, influyen en la manera como se diseñan los puestos, los procesos de trabajo, generando una red de relaciones para la transferencia del conocimiento por toda la organización” (Rodríguez, 2013, p.1), la gestión del conocimiento en el contexto universitario, como estructura facilita la optimización del recurso, la capacidad, para un mejor desempeño del trabajo colaborativo y la cultura organizacional. Por tanto, el conocimiento aportado por la institución de educación superior, tiene como intención beneficiar al ser humano, la industria, el gobierno o para la sociedad, ayuda al capital intelectual en los procesos de generación y difusión del conocimiento que impulsa el avance científico.

\section{MATERIALES Y MÉTODOS}

En referencia a este apartado, la investigación se fundamentó en el paradigma cualitativo, bajo el método de Investigación Acción Participante, lo cual tuvo como propósito, establecer la calidad total como alternativa de gestión para el desarrollo de la producción científica en la educación superior, "Estudia el lado subjetivo de la vida de modo cómo las personas se ven a sí mismas y cómo perciben su entorno, buscan describir el sentido y el significado de los fenómenos objeto de estudio" (Martínez, 2013, p.35), este paradigma permitió abordar la problemática desde su naturalidad, tomó en cuenta a los docentes investigadores de las instituciones universitarias, involucradas en el hecho, suministraron la información necesaria para determinar los hallazgo y conclusiones.

Las fases desarrolladas en la investigación, expresan para el desarrollo de la investigación acción participante, el diagnóstico, consiste en la preocupación que surge en el investigador para resolver el problema. La planificación, se refiere a las actividades y estrategias que el investigador desarrollara durante en el estudio sustentado en el diagnóstico (Hurtado y Toro, 2000). Sin embargo, los informantes de la investigación, son aquellas personas que suministran datos relacionados con objeto de estudio, "es un método antropológico estándar ampliamente usado en las investigaciones y este término se aplica a cualquier persona que pueda brindar información detallada debido a su experiencia o conocimiento de un tema específico" (Hernández, Fernández y Baptista 
2010, p. 21).

En consecuencia, el procedimiento empleado para seleccionar a los informantes, fue el muestreo no probabilístico de tipo intencional, la técnica utilizada es la observación directa, representada mediante el instrumento de guion de observación, dirigido a cincuenta (50) docente universitarios con la finalidad de recolectar información de la situación actual sobre la gestión de producción científica. De igual manera, se empleó como técnica la entrevista y como instrumento la guía de entrevista estructurado en quince preguntas, (15) dirigida a los diez (10) docentes investigadores en instituciones universitarias de educación superior. La validez de la Investigación se fundamentó en la técnica de la triangulación "las intersecciones o coincidencias partiendo de diferentes apreciaciones, fuentes informáticas, o puntos de vista con respecto a un mismo fenómeno" (Tamayo, 2009, p.80).

Desde esta concepción, se logró contrastar la información mediante la teorización de los datos y opiniones obtenidas de los informantes implicados. Durante el proceso de la investigación se empleó como técnica cualitativa la triangulación, categorización y teorización de los datos. La Fiabilidad de la investigación "consiste en solicitar la colaboración de los sujetos informantes para constatar la objetividad de la información recabada" (Martínez, 2013, p. 200). Al respecto, la fiabilidad facilitó verificar a futuro que la información recolectada de los informantes coincida con los resultados obtenidos por el investigador, es consistente con lo que ven o dicen los sujetos seleccionados.

\section{ANÁLISIS DE RESULTADOS Y DISCUSIÓN}

El siguiente apartado, presenta los hallazgos y discusiones del estudio fundamentado en las técnicas cualitativas de la categorización y teorización, tomando como base las opiniones de los informantes, el diagnóstico que sustenta la acción participante cuyos resultados se obtuvieron de la entrevista aplicada a los diez docentes investigadores (Martínez, 2013, p. 240). A continuación, se presenta un ejemplo, las dos (02) primeras preguntas de la entrevista: 
Tabla 1. Categorización de las opiniones obtenidas de los informantes de la investigación.

Objetivo general: Implementar acciones formativas referentes a la calidad total como medio de gestión para la producción científica en las instituciones universitarias de la provincia Imbabura del Ecuador durante el año 2017.

Unidad de análisis

Calidad Total

\section{Subcategorías}

Gestión de la producción científica.

\section{Categorización de la información}

Respuesta del instrumento aplicado al docente

\section{Pregunta 1. ¿Qué entiende por} gestión de la producción científica?

1. Manifestó que la gestión de la producción es responsabilidad de la institución.

2 Opinó que solo es un concepto referido a las ciencias administrativas.

3 La gestión de la producción no tiene que ver con el área científica.

4 Expresó que la gestión es un principio de contabilidad económica.

5 Opinó que la gestión está relacionada con la calidad y producción científica.

6 Exteriorizó que la gestión es un elemento que mejora los procesos de generación de conocimientos.

7 La gestión está referida a la calidad humana en los procesos.
Pregunta 2. ¿Cuáles son las herramientas para la gestión de la producción científica?

1 Las herramientas de la gestión en la administración es la comunicación asertivas.

2. Opinó que solo es un concepto complejo.

3. La gestión de la producción no tiene herramientas.

4. Diagrama de Pareto, diagrama de causa - efecto, Histograma.

5. Exteriorizó que ayudan a la resolución de conflictos.

6. Diagrama de flujo, Estatificación Estadística.

7. La gestión está referida como una sola herramienta en los procesos de calidad.

8 Opinó que la gestión contribuye significativamente en el proceso de producción científica de la institución.
8. Opinó que son diagrama de pareto, diagrama de causa - efecto, 


\begin{tabular}{lll}
\hline & $\begin{array}{l}\text { histograma, diagrama de causa - } \\
\text { efecto. }\end{array}$ \\
\hline 9 Desconoce el concepto de gestión \\
$\begin{array}{l}\text { aplicado a la producción científica. } \\
10 \text { Expresó que la gestión debe estar } \\
\text { vinculada a los procesos de } \\
\text { producción científica. }\end{array}$ \\
$\begin{array}{l}\text { Fuente: López, } 2017 . \\
\text { que ver con el área científica. }\end{array}$
\end{tabular}

Tabla 2. Teorización de las opiniones obtenidas de los informantes de la investigación.

Objetivo General: Implementar acciones formativas referentes a la calidad total como medio de gestión para la producción científica en las instituciones Universitarias de la provincia Imbabura del Ecuador durante el año 2017.

\begin{tabular}{|c|c|c|}
\hline \multicolumn{2}{|r|}{ Unidad de análisis } & Categorías \\
\hline \multicolumn{2}{|r|}{ Calidad Total } & iestión de la $p$ \\
\hline \multicolumn{3}{|c|}{ Teorización de la información } \\
\hline \multicolumn{2}{|r|}{$\begin{array}{c}\text { Pregunta 1. ¿Qué entiende por } \\
\text { gestión de la producción científica? }\end{array}$} & $\begin{array}{l}\text { Pregunta 2. ¿Cuáles son las } \\
\text { herramientas para la gestión de la } \\
\text { producción científica? }\end{array}$ \\
\hline $\begin{array}{c}1,5 \\
6,8 \\
10\end{array}$ & $\begin{array}{l}\text { Gutiérrez, Cárdenas, Hernández, y } \\
\text { Rivera (2006) la gestión de la } \\
\text { producción científica, está } \\
\text { relacionada con la nueva } \\
\text { universidad, puede beneficiarse de } \\
\text { un enfoque integrado de la gestión, } 1,7 \text {, } \\
\text { el conocimiento, la ciencia y la } \\
\text { innovación pueden multiplicar las } \\
\text { oportunidades para favorecer el } \\
\text { desarrollo científico, sostenible y } \\
\text { humano. }\end{array}$ & $\begin{array}{l}\text { García (2016) las herramientas de } \\
\text { gestión de la calidad total, se } \\
\text { entiende por la aplicación de } \\
\text { principios fundamentados para la } \\
\text { resolución de problemas empíricos } \\
\text { como objetos de investigación. De } \\
\text { allí, como norma general el uso del } \\
\text { Histograma, Diagrama de } \\
\text { Dispersión, Estratificación, } \\
\text { Gráficos de Control, para establecer } \\
\text { la calidad en la producción } \\
\text { científica. }\end{array}$ \\
\hline $\begin{array}{l}2,3 \\
\text { y } 9 .\end{array}$ & $\begin{array}{l}\text { Garbanzo-Vargas (2016) la gestión } \\
\text { de la producción científica, además } \\
\text { está inmersa en el campo }\end{array}$ & $\begin{array}{l}\text { Guerrero \& Franco (2016). Las siete } \\
\text { herramientas básicas de calidad, es } \\
\text { una clasificación que responde a }\end{array}$ \\
\hline
\end{tabular}




\begin{tabular}{l|l|l}
\hline & $\begin{array}{l}\text { administrativo, también está } \\
\text { vinculada con la gestión del } \\
\text { conocimiento, el desarrollo } \\
\text { inteligente, desarrollo organizacional } \\
\text { para lograr la innovación y el } \\
\text { desarrollo tecnológico }\end{array}$ & $\begin{array}{l}\text { un conjunto de métodos gráficos } \\
\text { para identificar la solución de } \\
\text { problemas enfocados para la } \\
\text { mejora de los procesos, es decir, } \\
\text { progreso de la producción científica } \\
\text { en la institución universitaria. }\end{array}$ \\
\hline $\begin{array}{l}\text { Palacios, Quiñones, Caridad, Candó } \\
\text { ytros, (2016) expresan que la } \\
\text { gestión no solo está referida a un } \\
\text { principio administrativo, de } \\
\text { contabilidad o proceso humano, sino } \\
\text { que la Gestión en la investigación 4, 6, } \\
\text { científica propicia información y 8 } \\
\text { nuevos conocimientos en el saber y } \\
\text { hacer, para la iniciación de } \\
\text { actividades investigativas que } \\
\text { ayudan a profundizar el desarrollo } \\
\text { humano y tecnológico. }\end{array}$ & $\begin{array}{l}\text { Talavera (2012), manifiesta que las } \\
\text { siete herramientas básicas de la } \\
\text { calidad, son descritas como } \\
\text { métodos para la mejora continua y } \\
\text { la de problemas, ellas son: } \\
\text { Comprobación, Gráficos de Control, } \\
\text { Histograma, Diagrama de Pareto, } \\
\text { Diagrama de Dispersión y la } \\
\text { Estratificación. }\end{array}$ \\
\hline
\end{tabular}

En correlación con el primer objetivo específico, diagnosticar el nivel de producción científica existente en las instituciones Universitarias de la provincia, se comprobó que la mayoría de los docentes desconocen el significado que representa la gestión para fortalecer la producción científica, al respecto la gestión de la producción científica, está vinculada con un enfoque integrado, transdisciplinario de pensamiento complejo que facilita la generación de nuevos conocimientos, la innovación, la ciencia para aumentar las oportunidades que favorecer el desarrollo científico y humano (Gutiérrez, Cárdenas, Hernández, y Rivera, 2006).

Además, se logró evidenciar que un grupo de docentes consideró que la gestión solo está vinculada a las ciencias administrativas y no tiene pertinencia con la producción científica de la institución universitaria, la gestión de la producción científica, está introducida en el campo administrativo, pero al mismo tiempo está vinculada con la gestión del conocimiento, el desarrollo inteligente, desarrollo organizacional que facilita a la institución lograr la innovación y el desarrollo tecnológico enmarcado en las 
herramientas de la calidad total. Estas herramientas permiten diagnosticar problemas mediante la interacción entre el sujeto y objeto de estudio para generar conocimiento y nuevos aportes (Garbanzo-Vargas, 2016).

Otro de los aspectos que se presentó, fue que la gestión es considerada como un principio de contabilidad económica y a la calidad humana en los procesos. En efecto, la gestión de la producción científica, no solo concerniente a un principio administrativo o proceso humano, sino que la favorece la generación de nuevos conocimientos, contribuye a profundizar el desarrollo humano y tecnológico en el ámbito universitario (Palacios, Quiñones, Caridad, Candó y otros, 2016).

En función de las respuestas obtenidas de la primera pregunta de la entrevista aplica a los docentes, se muestra como ejemplo que la mayoría de los encuestados la gestión aplicada hacia la generación de nuevos conocimientos, la ciencia, y la epistemología, lo cual incide en la problemática, el bajo nivel de producción científica existente en las instituciones universitarias de la provincia de Imbabura, Ecuador.

Tabla 3: Planificación de las Acciones.

Desarrollar acciones vinculadas con la calidad total como alternativa para la gestión de la producción científica en la educación superior durante el año 2017.

\begin{tabular}{|c|c|}
\hline ACCIÓN & OBJETIVO ESPECÍFICO \\
\hline $\begin{array}{l}\text { Acción } 1 \\
\text { Reunión socializadora con directores de } \\
\text { investigación y docente de las } \\
\text { instituciones universitarias de la } \\
\text { provincia Imbabura. }\end{array}$ & $\begin{array}{l}\text { Presentar las acciones formativas a los } \\
\text { directores de investigación mediante una } \\
\text { reunión para el fortalecimiento de la gestión } \\
\text { en la producción científica universitaria. }\end{array}$ \\
\hline \multirow{2}{*}{$\begin{array}{l}\text { Acción } 2 \\
\text { Explicar el uso de la calidad total para la } \\
\text { gestión de la producción científica en la } \\
\text { institución universitaria. }\end{array}$} & $\begin{array}{l}\text { Realizar un taller sobre la Calidad Total y } \\
\text { sus herramientas que faciliten la gestión de } \\
\text { la producción científica. }\end{array}$ \\
\hline & $\begin{array}{l}\text { Efectuar ejercicios prácticos sobre la } \\
\text { utilización de las herramientas de la calidad } \\
\text { total que ayuden con la gestión para la } \\
\text { producción científica. }\end{array}$ \\
\hline
\end{tabular}




\begin{tabular}{|c|c|}
\hline $\begin{array}{l}\text { Acción } 3 \\
\text { Exponer los lineamiento de la ISO } 9001 \\
\text { como clave de la calidad total para la } \\
\text { gestión de la producción científica } \\
\text { universitaria mediante la planificación, } \\
\text { ejecución, control, monitoreo y medición } \\
\text { internacional. }\end{array}$ & $\begin{array}{l}\text { Presentar mediante un taller los } \\
\text { lineamientos de la ISO } 9001 \text { como clave de } \\
\text { la calidad total para la gestión de la } \\
\text { producción científica universitaria a través } \\
\text { de la planificación, ejecución, control, } \\
\text { monitoreo y medición internacional. }\end{array}$ \\
\hline $\begin{array}{l}\text { Acción } 4 \\
\text { Informar a los docentes los indicadores } \\
\text { establecidos por el CEAACES para la } \\
\text { gestión de la producción científica en las } \\
\text { instituciones universitarias. }\end{array}$ & $\begin{array}{l}\text { Realizar una charla con la participación de } \\
\text { un funcionario del CEAACES y docente } \\
\text { investigadores de las universidades que } \\
\text { facilite la gestión de la producción } \\
\text { científica. }\end{array}$ \\
\hline $\begin{array}{l}\text { Acción } 5 \\
\text { Expresar a los docentes la utilidad de la } \\
\text { base datos de Scimago Journal \& } \\
\text { Country Rank, Elsevier, ISI Wed of } \\
\text { Knowledge para la gestión de la } \\
\text { producción científica. }\end{array}$ & $\begin{array}{l}\text { Explicar el uso de la base datos de } \\
\text { Scimago Journal \& Country Rank, Elsevier } \\
\text {, ISI Wed of Knowledge mediante una } \\
\text { exposición que permita la gestión de la } \\
\text { producción científica }\end{array}$ \\
\hline $\begin{array}{l}\text { Acción } 6 \\
\text { Exponer la importancia de la utilización } \\
\text { de los textos científicos como medios } \\
\text { que contribuyen con la gestión en la } \\
\text { producción científica }\end{array}$ & $\begin{array}{l}\text { Presentar la importancia de la utilización de } \\
\text { los textos científicos tales como; artículos, } \\
\text { ensayos, capítulos de libros mediante una } \\
\text { exposición que contribuya con la gestión de } \\
\text { la producción científica. }\end{array}$ \\
\hline
\end{tabular}

Fuente: López, 2017.

En referencia a la planificación de las acciones fundamentadas en la calidad total como medio de gestión para la producción científica en las instituciones universitarias, la primera acción, titulada "Reunión socializadora con directores de investigación y docente de las instituciones universitarias de la provincia Imbabura del Ecuador". Se ejecutó de la siguiente manera: el inicio, consistió en establecer contacto directo con las personas involucradas con la temática objeto de estudio a fin de presentar un cronograma de acciones destinado a mejorar la gestión de la producción científica. De allí, el desarrollo de la acción permitió explicar la utilización de las herramientas de la calidad total, los lineamientos de la ISO 9001 como clave de la calidad total, los 
indicadores establecidos por el CEAACES para la gestión de la producción científica, el uso de la base de datos de Scimago Journal \& Country Rank, Elsevier, ISI Wed of Knowledge y la importancia de la utilización de los textos científicos que contribuyen con la gestión en la producción científica.

La evaluación de esta acción formativa, se llevó a cabo mediante la aplicación del instrumento de escala de estimación que permitió verificar la aprobación de las acciones formativas, por parte de los directores de investigación de las instituciones universitarias de la provincia Imbabura del Ecuador. Así como también, se logró evidenciar como indicador, reconocer la calidad total como medio de gestión que garantice la producción científica en las instituciones universitarias. Por último, como indicador, se pudo valorar mediante las acciones formativas los lineamientos establecidos por el CEAACES para la calidad total y la gestión de la producción científica.

\section{CONCLUSIONES}

El abordaje de la temática referente a la calidad total como medio de gestión para la producción científica en la educación superior se fundamentó en una investigación tipo cualitativa, bajo la modalidad de Investigación Acción Participativa. Por consiguiente, el diagnóstico obtenido sobre el nivel de la producción científica permitió establecer acciones como para fortalecer y mejorar la producción científica en las instituciones universitarias mediante el uso de las herramientas de calidad total para llevar a cabo una mejor gestión del conocimiento.

Además, se logró valorar la importancia de los indicadores de calidad establecidos por el CEAACES mediante la publicación de texto en base datos de Scimago Journal, Country Rank, Elsevier, que contribuye a elevar la producción científica. De ahí, "La producción científica, es considerada como la parte materializada del conocimiento generado, es más que un conjunto de documentos almacenados en una institución de información, se considera también, contempla todas las actividades académicas y científicas de un investigador (Salomón y Martínez, 2007, p. 33).

Por consiguiente, las herramientas de la calidad total, tales como: Diagrama Causa Efecto, Gráficos de Control Hoja de Comprobación, Diagrama de Dispersión Histograma, Diagrama de Pareto y la Estratificación que facilitaron la búsqueda 
intencionada de saberes, combinada con criterios de observación, clasificación, análisis, para establecer contacto con la realidad para formular nuevas teorías, modificar las existentes y a su vez, generar un mayor impacto en la producción científica institucional.

Finalmente, reconocer la calidad total como medio de gestión para valorar los lineamientos establecidos por el CEAACES en relación con la calidad total y la gestión de la producción científica a nivel de la educación superior.

\section{REFERENCIAS BIBLIOGRÁFICAS}

Camisón, C, Cruz y S. González, T. Gestión de la Calidad; Concepto, Enfoques, Modelos y Sistema. Consultado el 10 de enero de 2006. Recuperado de https://goo.gl/6zuVzY

Cardoso, E., y Cerecedo, M. (2012) Análisis de la producción científica de la Universidad Autónoma de Tamaulipas y evaluación de su impacto en los indicadores educativos de calidad. Consultado el 10 de enero 2017. Revista de la educación superior. Recuperado de https://goo.gl/XnPnqo

Carro, R. y González Gómez, D. (2012). Estrategia de producción/operaciones en un entorno global. México: Editorial McGraw Hill.

Consejo de Evaluación, Acreditación y Aseguramiento de la Calidad de la Educación Superior, (CEAACES) (2017) Evaluación de universidades categoría “D” y concluye una etapa histórica para la educación superior del país. Consultado el 25 de febrero de 2017. Recuperado de https://goo.g//9wqKt6

Ecuador, D. P., y Pazmiño Granizo, E. (2015). Rendición de Cuentas 2014.

El Comercio (2017). Instituto de Tecnología Massachusetts encabeza el ránking de las 1 000 mejores universidades del mundo; dos centros de Ecuador están en la lista, Consultado el 27 de febrero 2017. Recuperado de https://goo.gl/oFVKmw.

García, L. A. M. (2016). Indicadores de la gestión logística. Ecoe Ediciones. 
Guerrero, V., \& Franco, J. (2016). Diseño de un modelo de gestión de procesos utilizando herramientas BPM para mejorar la eficiencia del proceso de recaudación en la IEP ADEU Deportivo SAC, Chiclayo-2014.

Garbanzo-Vargas, G. M. (2016). Desarrollo organizacional y los procesos de cambio en las instituciones educativas, un reto de la gestión de la educación. Revista Educación, 40(1), 67-87.

Gutiérrez, D. H., Cárdenas, F. B., Hernández, Y. S., y Rivera, S. A. M. (2006). La nueva universidad cubana y su contribución a la universalización del conocimiento. La Habana: Editorial Félix Valera.

Hernández, S. Fernández, C. y Baptista, L. (2014). Metodología de la Investigación. México: McGraw Hill.

Hurtado, I. y Toro, J. (2005). Paradigmas y Métodos de Investigación en tiempos de cambio. Valencia, Venezuela. Episteme Consultores Asociados C. A.

Leisyte, L., \& Boer, H. D. (2009). The balance between teaching and research in Dutch and English universities in the context of university governance reforms. Higher Education, 58(5), 619-635.

Martínez, Migueles. (2013). Ciencia y Arte en la Metodología Cualitativa. México: Editorial Trillas México.

Molina, J. Gómez, J. Cordero, C. Barbosa, J. Pozo, E. (2015). Aportes y buenas prácticas para la investigación y la difusión de la ciencia universidad politécnica salesiana. Congreso Internacional: investigación, producción científica, 1-4 Abril (ponencia).

Palaciosl, O. S., Quiñonesll, L. C., de la Caridad, R., Candólll, B., MolinalV, M. M. S., SosaV, R. F., \& Cabrera, E. (2016) Gestión de la investigación científica en el posgrado de las especialidades en Ciencias Médicas Management of scientific research in the graduate specialties in Medical Sciences. 
Rodríguez, M. (2013). Generación y transferencia de conocimiento, Universidad Libre, seccional Cali. Cali, Colombia. Recuperado de https://goo.gl/dZQaKr

Salomón, Y. y Martínez, A. (2007). Producción Científica, Ciencias de la Información, Revista Redalyc, La Habana, Cuba. 38 (03) 33-34.

Talavera, C. (2012). Métodos y Herramientas de Mejora aplicados en la Administración Pública. Recuperado de https://goo.gl/ftw3TA

Tamayo, T. (2009). El proceso de la Investigación Científica. México: Editorial Limusa.

Universidad Autónoma de Madrid (UAM) (2017). Producción Científica de la UAM Gestión, consulta y difusión de la producción científica de la universidad. Consultado el 27 de febrero 2017. Recuperado de https://goo.gl/p6iDzj. 